\title{
The South Atlantic Anomaly drift on the proton flux data of satellite experiments
}

\author{
Aleksandrin S.Yu. \\ Author affiliation \\ National Research Nuclear University MEPhI (Moscow Engineering Physics Institute) \\ E-mail: SYAleksandrin@mephi.ru
}

\section{Galper A.M.}

Affiliation

National Research Nuclear University MEPhI (Moscow Engineering Physics Institute)

E-mail: AMGalper@mephi.ru

\section{Koldashov S.V.}

Affiliation

National Research Nuclear University MEPhI (Moscow Engineering Physics Institute)

E-mail: SVKoldashov@mephi.ru

\section{Zharaspayev T.R.}

\section{Affiliation}

National Research Nuclear University MEPhI (Moscow Engineering Physics Institute)

E-mail: masscry@gmail.com

\begin{abstract}
Dynamics of flux of high-energy charged particles trapped by geomagnetic field has been studied in this report. As is known Earth's magnetic field changes in direction and magnitude, therefore the South Atlantic Anomaly (SAA) region drifts. Geographical distributions of proton flux (80$100 \mathrm{MeV}$ ) were studied since 2007 to 2014 using data of the ARINA satellite experiment. The ARINA satellite experiment is designed to study the physics origin of high-energy charged particle variations and bursts. ARINA placed on Resurs-DK1 satellite, which launched in 2006 and is still operating. Detail analysis of the SAA drift was fulfilled. The SAA region $(L=1.15-2.2)$ was divided on zones with 0.05 step value. Maximum of proton flux was determined for each zone. Position of maximum drifts in west direction, which corresponds to the dynamics of the geomagnetic field. Average velocity of SAA longitude drift is almost independent of the L-shell and has a value about 0.5 degrees a year.
\end{abstract}

The 34th International Cosmic Ray Conference,

30 July- 6 August, 2015

The Hague, The Netherlands

\footnotetext{
* Speaker.
} 


\section{Introduction.}

The geomagnetic field plays an important role in the life of not only humanity but the entire living world on the Earth. Earth's magnetosphere has the function of a shield that protects all life from the harmful influence of solar radiation. Earth's magnetic field is known to reverse periodically during its existence. A geomagnetic reversal happens with period of about several hundred thousand years. Now the value of the geomagnetic field is decreasing rather fast and the next reversal have to be rather soon in historical point of view (less than 1000 years, some author consider that within a human lifetime) [1]. Life on the Earth can remain undefended for that time. Magnetic field of the Earth studies by ground stations and satellite experiments. Magnetosphere of the Earth has dipole structure and can trap charged particles. These particles accumulate and form radiation belts. The axis of geomagnetic dipole is inclined to the axis of rotation of the Earth and is shifted from its center. Therefore, nowadays the radiation belt descends closest to the Earth's surface in Brazil and satellites, passing through this area are recording large fluxes of charged particles. This region is named the South Atlantic Anomaly (SAA). So we can study change of the geomagnetic field using charged particle data of satellite experiments. That investigation has been done in previous work [2] using data of three different satellite experiments which were launched during 20 years. The velocity of the SAA drift was about $0.41 \mathrm{grad} /$ year in western direction.

This report analyzes the data of satellite experiment ARINA which placed on board the Russian satellite Resurs-DK1. The advantage of this approach is that the experiment has been going on nearly nine years and it is possibility to observe the dynamics of particle fluxes over a long period with the same instrument.

\section{Instruments.}

The ARINA instrument is scintillation spectrometer. It is designed as a pyramid of scintillation layers. Each of the layers viewed in two photomultiplier tubes. In order to determine the energy of the detected particle, has considered those one, which stopped at the detector, ie the lower layer is used as anticoincidence detector. This determines the energy range of the detected particles. For electrons it is 3-30 MeV for protons - 30-100 MeV. In this analysis we used the channel that recorded proton flux with energies from 80 to $100 \mathrm{MeV}$. The ARINA on board Resurs-DK1. It was launched in 2006 to orbit with an inclination of 70 degrees and an altitude of 350-600 km The ARINA spectrometers developed by the MEPhI.

The instrument acceptance is about $10 \mathrm{~cm}^{2} \cdot s r$. The physics scheme and performances of the instrument are described in detail in [3].

\section{Experimental data.}

Analysis of the SAA drift was done in previous work using data of three satellite experiments. Statistics of this experiments were not high, and the analysis concerned only with a drift of the maximum of the particle flux. Experiment ARINA has a much greater acceptance and statistics has increased in comparison with previous experiments. This allows to fufill analysis for the selected $L$-shells. A distribution of proton flux $\left(E_{p}\right.$ is $\left.80-100 \mathrm{MeV}\right)$ is shown in fig. 1 . The SAA region situates where flux of particles increases thousands of times. 


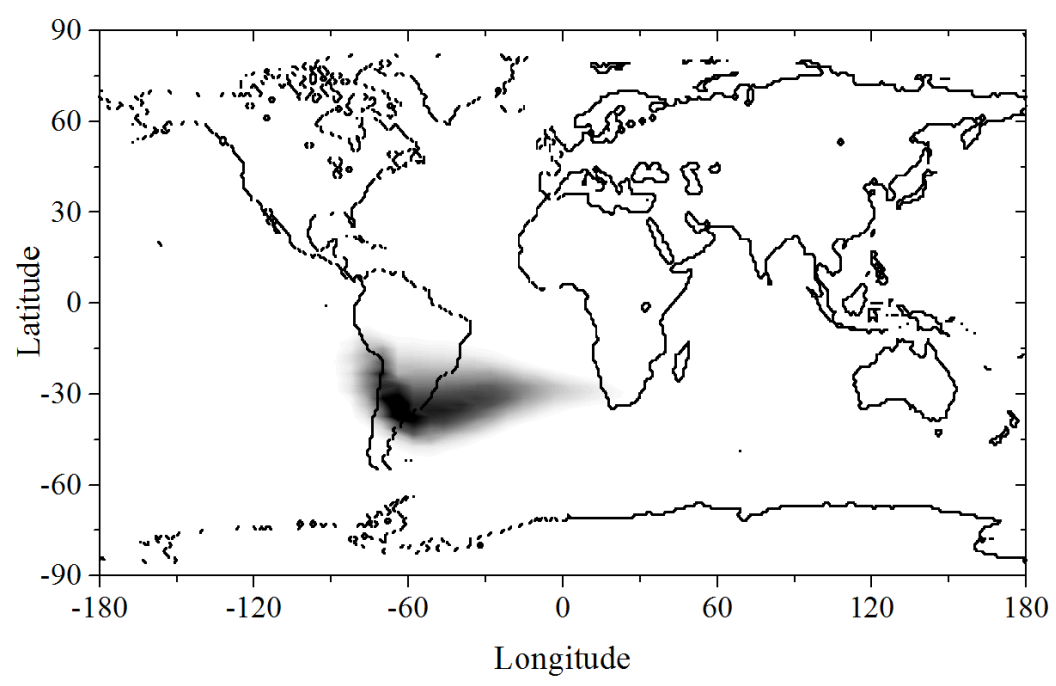

Figure 1: Proton flux geographic distribution $\left(E_{p}\right.$ is $\left.80-100 \mathrm{MeV}\right)$.

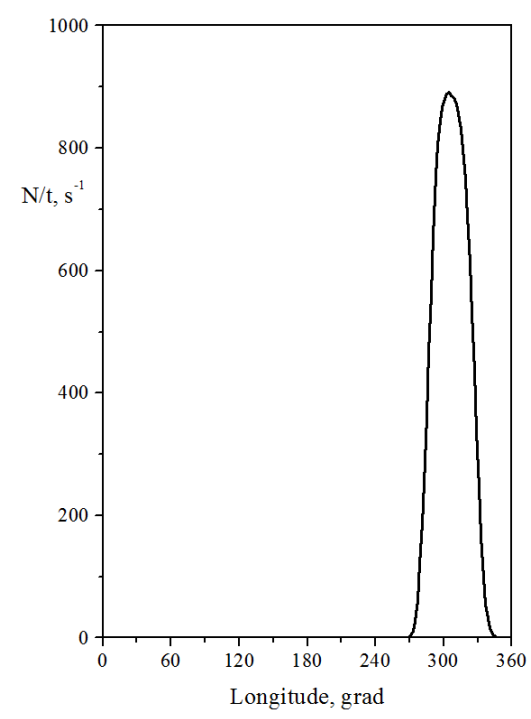

Figure 2: Proton flux along $L=1.2$.

To analyze the dynamics of high-energy proton flux a separate $L$-shell was selected and defined particle flux along it, depending on the longitude. Figure 2 shows this dependence for the $L$-shell 1.2. The maximum of proton flux are observed in the region of the SAA as seen in the figure.

Dynamics of high-energy proton flux in the SAA region has been analyzed. Maximum flux at selected shell moves to the west. The average rate of it is about $0.45 \pm 0.07 \mathrm{grad} /$ year for $L=1.2$ and $0.54 \pm 0.1 \mathrm{grad} /$ year for $L=1.5$. (fig. 3).

\section{Model of geomagnetic field.}

The motion of charged particles in near-Earth space defined by the magnetic field of the Earth, so that the position of the South anomaly is determined by the geomagnetic field.The analysis of the 

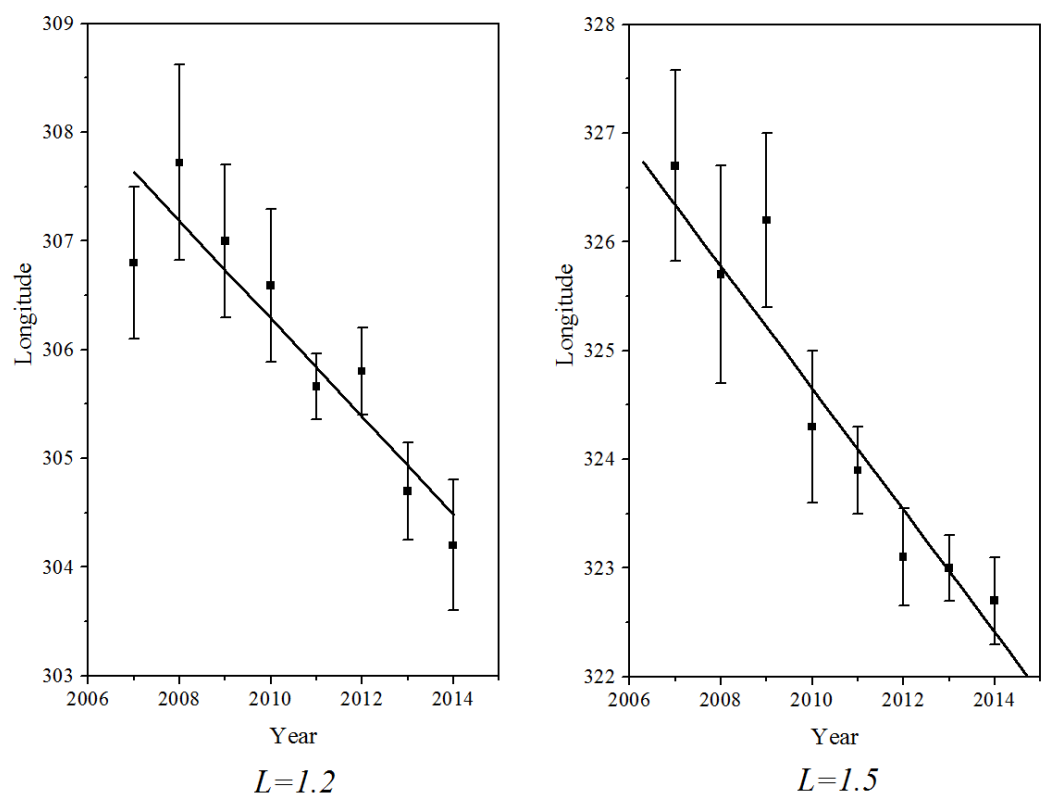

Figure 3: Drift of maximum of proton flux.

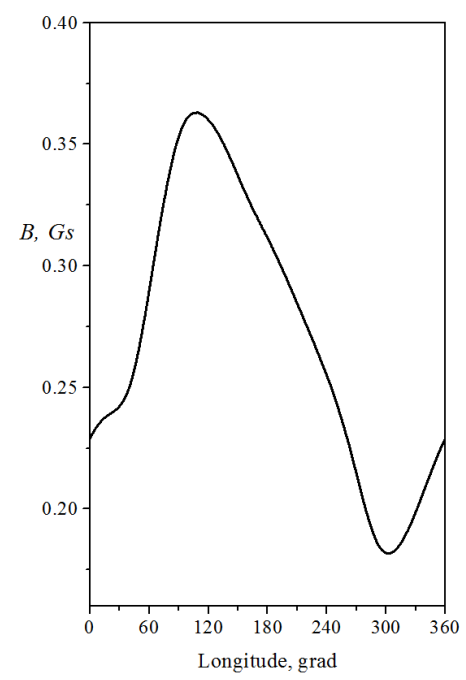

Figure 4: The dependence of the geomagnetic field on the longitude, $L=1.2$.

magnetic field value along the selected $L$-shell depending of longitude was conducted. The IGRF12 model of geomagnetic field was used in this work[ [4]. The dependence of the geomagnetic field on the longitude is shown in fig. 4. The value of magnetic field is minimal in the SAA region.

The analysis of location change of geomagnetic field anomalies according to the model from 2007 to 2014 shows that the SAA is drifting to the west with some speed. The average drift velocity anomaly according to the geomagnetic field was about $0.34 \pm 0.08 \mathrm{deg} / \mathrm{year}$ on $L=1.2$ and $0.36 \pm 0.09$ deg/year on $L=1.5$ for the selected period (fig. 5).

The SAA's drift velocity of geomagnetic model and data obtained with analysis of protons flux agree within error. The difference between the position of the maximum flux and minimum values 

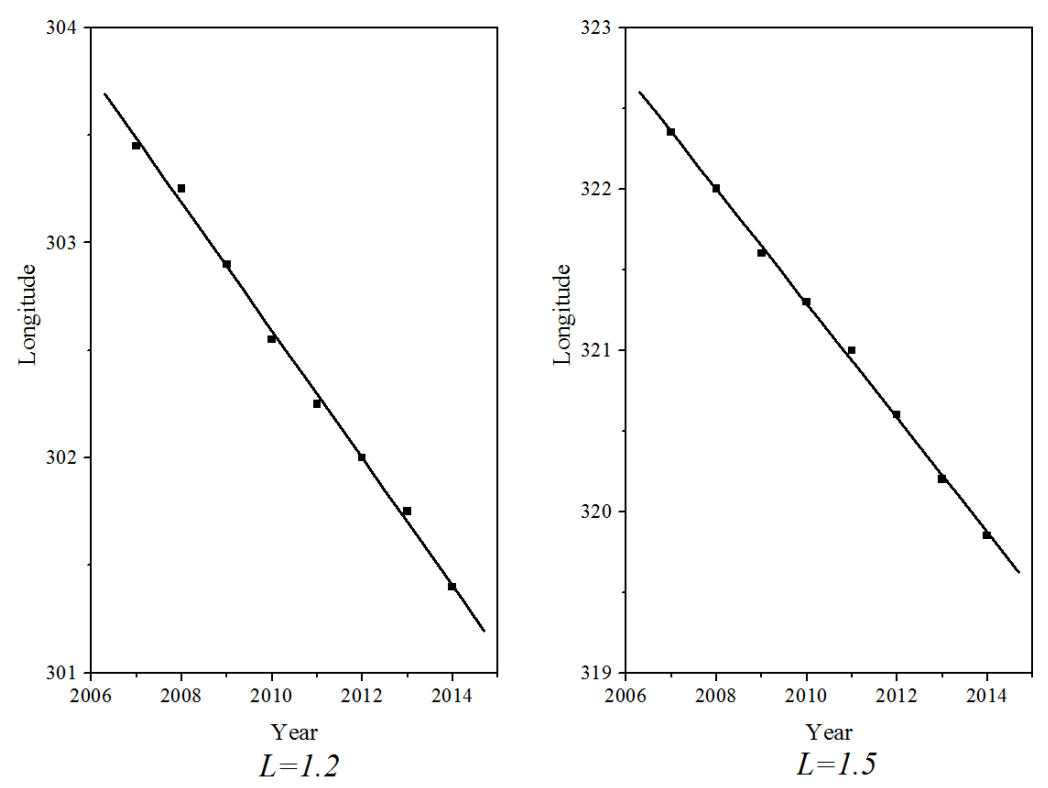

Figure 5: The drift of the SAA by the IGRF model data.

of the geomagnetic field observed apparently due to the fact that the orientation of the device in the SAA is not exactly perpendicular to the magnetic field lines and particle flux in this region is strongly inhomogeneous.

\section{Conclusion.}

The analysis of the dynamics of the SAA was carried out in this report. It has been shown that, according to the proton flux drift velocity in the western direction was $0.45 \pm 0.07 \mathrm{grad} / \mathrm{year}$ for $L=1.2$ and $0.54 \pm 0.1 \mathrm{grad} /$ year for $L=1.5$ during the period from 2007 to 2014 . According to the model of Earth's magnetic field the SAA drifts at a rate of $0.34 \pm 0.08 \mathrm{deg} / \mathrm{year}$ on $L=1.2$ and $0.36 \pm 0.09 \mathrm{deg} /$ year on $L=1.5$, which is consistent with the data of proton flux.

\section{References}

[1] Leonardo Sagnotti, Giancarlo Scardia, Biagio Giaccio, Joseph C. Liddicoat, Sebastien Nomade, Paul R. Renne, Courtney J. Sprain; (21 July 2014) "Extremely rapid directional change during Matuyama-Brunhes geomagnetic polarity reversal". Geophysical Journal International V199 I.2: pp.1110âĂŞ1124

[2] A.M. Galper, B.I. Luchkov. About Brazil anomaly drift. Geomagnetizm I Aeronomiya, 1983. T.23. No.4. S.669 (in Russian).

[3] Bakaldin A.V. Batishchev A.G., Voronov S.A. and others. "The ARINA satellite experiment to study the seismic effects in the flux of high-energy particles in the magnetosphere of the Earth".

Cosmicheskie Issledovaniya 2007, T 45, âĎÚ5, p. 471. (in Russian).

[4] http://www.ngdc.noaa.gov/IAGA/vmod/index.html 